Cahiers $d u$ MONDE RUSSE

\section{Cahiers du monde russe}

Russie - Empire russe - Union soviétique et États indépendants

$45 / 3-4 \mid 2004$

Varia

\title{
François-Xavier Nérard, Cinq pour cent de vérité
}

\section{Tamara Kondratieva}

\section{OpenEdition \\ Journals}

Édition électronique

URL : https://journals.openedition.org/monderusse/4201

DOI : 10.4000/monderusse.4201

ISSN : $1777-5388$

\section{Éditeur}

Éditions de l'EHESS

\section{Édition imprimée}

Date de publication : 1 juillet 2004

Pagination : 696-698

ISBN : 2-7132-2009-2

ISSN : $1252-6576$

\section{Référence électronique}

Tamara Kondratieva, « François-Xavier Nérard, Cinq pour cent de vérité », Cahiers du monde russe [En ligne], 45/3-4 | 2004, mis en ligne le 03 juin 2009, consulté le 03 septembre 2022. URL : http:// journals.openedition.org/monderusse/4201 ; DOI : https://doi.org/10.4000/monderusse.4201

Ce document a été généré automatiquement le 3 septembre 2022

Tous droits réservés 


\title{
François-Xavier Nérard, Cinq pour cent de vérité
}

\author{
Tamara Kondratieva
}

\section{RÉFÉRENCE}

François-Xavier NÉRARD, Cinq pour cent de vérité. La dénonciation dans l'URSS de Staline (1928-1941). Paris, Tallandier, 2004, 533 p.

Les dernières avancées de la recherche sur l'histoire de l'URSS ont déjà fait voler en éclats beaucoup de préjugés idéologiques, y compris le schématisme et l'extrémisme des courants « totalitariste » et « révisionniste ». Le livre de François-Xavier Nérard représente, lui aussi, une contribution importante dans ce sens en ce qu'il offre une étude approfondie de réalités complexes et permet l'objectivation des données puisées dans des archives nouvellement ouvertes (les fonds des Archives centrales moscovites de l'État-GARF, de l'économie-RGAE, du parti-RGASPI, des organisations de jeunesse-CHDMO et de la Défense-RGVA, mais également ceux de province, à Saratov-GASO, CHDNISO et à Nijni-Novgorod-GANO, GOPANO). Il démolit l'image sommaire du délateur méprisable et anonyme glissant à la police politique un petit mot qui pourra être fatal pour un proche. À sa place, ainsi qu'à celle de toutes les idées reçues sur la délation dans l'URSS de Stalin, F.-X. Nérard fait découvrir un phénomène relevant de l'anthropologie historique et de la culture politique séculaire. Ainsi des centaines de milliers de Soviétiques s'adressaient non pas tant à la police secrète qu'à un réseau important et multiforme d'instances (Inspection ouvrière et paysanne, journaux, bureaux des plaintes, etc.) susceptibles de saisir l'expression du mécontentement ou spécialement instituées à cet effet. Dans de longues lettres, ils exprimaient leurs doléances sur les conditions de vie, de travail (incluant des critiques à l'égard de la direction), les rapports de voisinage, les problèmes liés aux services publics. Rarement anonymes, ces lettres mettaient en avant des défauts dans le fonctionnement du monde professionnel, des injustices et des situations insupportables au quotidien. Selon le langage de l'époque, leurs auteurs envoyaient des «signaux » aux autorités dans le but d'attirer leur attention sur ce qui n'allait pas dans la vie publique ou privée. Cependant ils 
s'abstenaient, à de rares exceptions près, de critiquer le pouvoir central. C'était tout le contraire : en manifestant ainsi leur confiance au régime, ils espéraient que les autorités prendraient des mesures contre des comportements déviants de voisins, chefs, collègues, amis ou parents.

Peut-on dire qu'en engageant cette action, bien intentionnée dans la plupart des cas et à maintes reprises encouragée d'en haut, les Soviétiques dénonçaient leurs proches? S'agit-il de délation stricto sensu ? L'auteur propose de distinguer la délation moralement condamnable, qui vise à nuire à un individu, et la dénonciation au sens plus large, qui révèle au pouvoir des dysfonctionnements, des abus et des déviations. Le projet de l'ouvrage est de démontrer que la spécificité du phénomène en URSS, à la différence de la France pendant la Révolution ou l'Occupation, de l'Italie fasciste, de l'Allemagne nazie ou des États-Unis à l'époque du maccarthysme, consiste dans «l'irréductibilité de la dénonciation à la délation ».

Pour commencer, dans la première partie, intitulée "Protester sous Staline ", Nérard rappelle les origines d'une pratique de la révélation au pouvoir qui remonte aux débuts $\mathrm{du}$ règne des tsars, se perpétue durant des siècles et est utilisée par le souverain comme par ses sujets au nom de leur union sacrée. À la fin des années 1920, l'adresse au pouvoir est particulièrement encouragée au cours de la première campagne d'autocritique lancée en 1928. Stalin affirme alors qu'une critique ne révélant que $5 \%$ de vérité est de toute façon utile. Propagande, purges du parti, procès publics et répressions des années 1930 incitent le citoyen à être vigilant, à signaler au pouvoir les maux du premier pays socialiste en construction ainsi que les mauvaises actions et les noms (sic) de ses ennemis. L'image pieuse du peuple uni à l'État-parti est conservée, tout comme les illusions des protagonistes. La pratique du signal perdurera jusque dans les dernières années de l'Union soviétique.

4 L'intérêt et la force du livre résident en ce que l'auteur parvient à pénétrer au cœur des relations entre État-parti et population. Dans la deuxième partie (« La plainte: une pratique encouragée par le pouvoir»), il montre comment le "pouvoir réaffirme la nécessité de lui faire connaître tout ce qui ne va pas ». Le discours officiel présente le fait de pouvoir écrire à l'État comme «une des formes les plus importantes du développement de la démocratie ». Il tonne d'indignation envers la presse étrangère ou tous ceux qui voient dans cet échange entre l'État et les citoyens les marques de la délation. Les Soviétiques ne cessent de recevoir l'approbation officielle pour un acte qui consiste à prendre la plume afin d'écrire une lettre ou de faire une "déclaration » aux autorités. Avec une grande pertinence, Nérard avance l'idée d'un consentement de la population: «Même si c'est largement par absence de choix, celle-ci valide bien les structures mises en place par le pouvoir soviétique». L'historien passe en revue une grande quantité de matériaux (lettres, articles de presse, comptes rendus de réunions, discours, rapports d'inspection, etc.) et y débusque avec minutie les éléments permettant de comprendre comment s'élabore le consentement de l'individu à la pratique de la plainte, synonyme de dénonciation. Mais, ce faisant, les Soviétiques, dirigeants comme dirigés, maintiennent des relations dont ils revendiquent les valeurs civiques, morales et démocratiques. À l'époque, l'ensemble des discours produits, des lettres envoyées et des résultats obtenus (bien peu efficaces!) permet de maintenir l'image d'un pouvoir à l'écoute de son peuple. Une image fidèle aux canons du réalisme socialiste! Et c'est à Nérard que revient le mérite de jeter un éclairage nouveau sur toute cette fresque idyllique pour y montrer la dénonciation à l'œuvre. 
5 La troisième partie, intitulée « Le système au quotidien », et la quatrième, « Les signaux de la population », fournissent des réponses à de multiples interrogations concernant les motivations et les formes de la communication entre pouvoir et population. La dénonciation s'amplifie, ou se réduit à la délation, selon le contexte politique et les conditions de vie quotidiennes. On peut admettre avec l'auteur que, pendant la période étudiée (1928-1941), la délation se noie dans l'afflux des dénonciations qui marquent de leur empreinte le stalinisme. Nérard amène le lecteur à la conclusion que, pour les Soviétiques, c'était une forme d'expression du mécontentement et que «l'essentiel des racines du phénomène » résidait dans les «tensions sociales, la pénurie, la famine et la violence quotidienne ». La dénonciation aurait maintenu l'espoir, même minime, que la « voix d'un homme dans le désert » serait entendue.

6 En l'absence de statistiques globales disponibles dans les archives, l'approche phénoménologique semble justifiée dans le cas de ce livre. D'autant que la rigueur quantitative (nombre total de dénonciations? pourcentage de délation?) est rendue impossible par la nature du phénomène: les relations entre pouvoir et population en URSS sous Stalin se traduisent par une très grande ambiguïté non quantifiable. Grâce aux quelques statistiques accessibles et à la langue française, qui offre la possibilité de distinguer entre délation et dénonciation, l'auteur parvient à lever l'essentiel de cette ambiguïté. Une même finesse d'opération serait-elle possible en russe ? À la différence des Français, les Russes ne disposent que d'un seul mot, « délation » (donos), qui choque par la force de son sens très négatif et rend simpliste l'appréhension du phénomène. 